\title{
Simplified Heterocyclic Analogues of Fluoxetine Inhibit Inducible Nitric Oxide Production in Lipopolysaccharide-Induced BV2 Cells
}

\author{
Ju-Young Park, ${ }^{a}$ Seung-Woo Kim, ${ }^{b}$ Ja-Kyeong LeE, ${ }^{b}$ Weon Bin Im, ${ }^{a}$ Byung Kwan $\mathrm{JIN}_{,}{ }^{c}$ and \\ Sung-Hwa YooN*,a \\ ${ }^{a}$ Department of Molecular Science and Technology, Ajou University; San 5, Woncheon, Yeongtong, Suwon 443-749, \\ Republic of Korea: ${ }^{b}$ Department of Anatomy, Inha University; 3rd St. 7-241, Jung, Shinheung, Inchon 400-712, Republic \\ of Korea: and ${ }^{c}$ Department of Biochemistry \& Molecular Biology, Neurodegeneration Control Research Center, School of \\ Medicine Kyung Hee University; 1 Hoegi, Dongdaemoon, Seoul 130-701, Republic of Korea. \\ Received August 12, 2010; accepted January 13, 2011; published online January 20, 2011
}

\begin{abstract}
A series of fluoxetine, where the $N$-methylamino group was replaced and then simplified, were synthesized and their inhibitory effect was tested for nitric oxide (NO) production and inducible NO synthase (iNOS) expression in lipopolysaccharide (LPS)-induced BV2 cells. Although the synthesized compounds generally revealed weaker activity or greater cytotoxicity than fluoxetine, compound $10 \mathrm{a}$, in which the $N$-methylamino group in fluoxetine was replaced by morpholine, and the trifluoromethylphenyl ring was substituted with simple oxo group, suppressed NO production dose-dependently at 10, 20 and $40 \mu \mathrm{m}$ concentrations with less cytotoxicity than fluoxetine, and inhibited iNOS mRNA and protein expression at the same concentrations in LPS-induced BV2 cells. The results suggested that the trifluoromethylphenyl ring moiety in fluoxetine is not necessary for the suppression of NO production and that 10a has the potential as a potent inhibitor of NO production.
\end{abstract}

Key words fluoxetine analogue; microglia; BV2 cell; nitric oxide; inducible nitric oxide synthase

Microglial cells are a type of glial cell that acts as the first and main form of active immune defense in the central nervous system, and become activated by stimuli such as infection and injury. ${ }^{1)}$ The activation of microglia has been observed in neurodegenerative disease such as Alzheimer's disease (AD), Parkinson's disease, amyotrophic lateral sclerosis, multiple sclerosis (MS) and human immunodeficiency virusassociated dementia as the hallmark of brain inflammation. ${ }^{2,3)}$ Once activated, microglia release several inflammatory mediators such as reactive oxygen intermediates, nitric oxide (NO), interleukin $1 \beta$ (IL-1 $\beta$ ), interferon $\gamma$ (IFN- $\gamma$ ) and tumor necrosis factor alpha (TNF- $\alpha$ ), as well as neurotoxic substances. ${ }^{4,5}$ These mediators are thought to be accountable for brain diseases including ischemia, $\mathrm{AD}$ and neuronal death. ${ }^{6}$ Therefore, regulating microglial activation or suppressing these proinflammatory mediators could be a potential therapeutic approach to reducing the progression of these neurodegenerative disease. ${ }^{7)}$

Although intrinsic molecules of microglia activation have not yet been identified, experimental inducers of microglia, including lipopolysaccharide (LPS), amyloid- $\beta$ and IFN- $\gamma$, are known. ${ }^{8,9)}$ LPS, an outer-membrane component of gramnegative bacteria, is induced in inflammatory responses such as activated macrophages and microglia, produces TNF- $\alpha$, IL- $1 \beta$ and NO, and is involved in immune responses and host defense. ${ }^{10)}$ Among these chemicals, NO produced by inducible NO synthase (iNOS) can act as a cytotoxic agent to the cells by reacting with superoxide anions to produce highly reactive peroxynitrite anions $\left(\mathrm{ONOO}^{-}\right)$in pathophysiologic state, ${ }^{11,12)}$ leading to oxidative damage in host tissue, modification of DNA bases, and disruption of enzyme function and structural proteins. ${ }^{13,14)}$ Therefore, the high level of NO produced by iNOS has been considered an indicator of cytotoxicity in inflammation, NO production is a critical step in numerous neurodegenerative disease, and reduced NO production is a promising therapeutic strategy for the reduction of neuronal cell injury or death in various neuroinflam- matory and neurodegenerative diseases. ${ }^{15-17)}$

Fluoxetine, the selective serotonin reuptake inhibitor that is widely used in clinical practice for the treatment of depression, obsessive-compulsive disorder, bulimia and panic disorder, ${ }^{18,19)}$ has been shown to exert antioxidant potential via reversal of oxidative damage by enhancing the in vivo antioxidant defenses and improving the cellular antioxidant status following a stress-induced decline. ${ }^{20)}$ Fluoxetine has been found to have beneficial effects in animal models of stroke, MS, and epilepsy, even though fluoxetine has no positive effect in clinical studies with Parkinson's disease, AD and Huntington's disease. ${ }^{21)}$ Recently, we reported that fluoxetine exerts an anti-inflammatory effect by inhibiting nuclear factor $-\kappa \mathrm{B}(\mathrm{NF}-\kappa \mathrm{B})$ activity in the postischemic brain and NO in LPS-treated primary microglia. ${ }^{22)}$

In our ongoing efforts to find a potent inhibitor of NO production, a series of fluoxetine derivatives and analogues, where the $N$-methylamine group in fluoxetine was replaced by various heterocyclic amines, and then the trifluoromethylphenyl ring was simplified, were investigated in this study for their inhibitory activities on NO production and iNOS expression on BV2 cells stimulated with LPS.

\section{MATERIALS AND METHODS}

Instruments Melting points were determined on a Fisher-Johns melting point apparatus and were left uncorrected. ${ }^{1} \mathrm{H}$ - and ${ }^{13} \mathrm{C}$-NMR spectra were recorded on a Varian Gemini 400 spectrometer at $400 \mathrm{MHz}$ and $100 \mathrm{MHz}$, respectively. The chemical shifts given are relative to tetramethylsilane. Infrared spectra were recorded on a Nicolet 6700 Fourier transform (FT)-IR spectrometer. Electrospray ionization (ESI)-MS spectra were obtained by Shimadzu LCMS2010EV (Japan). Column chromatography was carried out using Merck silica gel 60 (230 - 400 mesh) (U.S.A.). All reactions were performed under a nitrogen atmosphere.

3-Chloro-1-phenylpropan-1-ol (3) To a solution of 3- 
chloro-1-propiophenone $2(25.0 \mathrm{~g}, 148 \mathrm{mmol})$ in methanol $(300 \mathrm{ml})$ was added sodium borohydride (16.8 g, $445 \mathrm{mmol})$. The mixture was stirred for $24 \mathrm{~h}$ at room temperature. After the reaction was complete, the solution was quenched with sodium dihydrogen phosphate $(66.7 \mathrm{~g}, 556 \mathrm{mmol})$. The solvent was removed by rotary evaporator and the residue was extracted with hexane and the organic layer was washed with water, dried over $\mathrm{Na}_{2} \mathrm{SO}_{4}$ and concentrated in vacuo to give the title compound as a colorless oil $(24 \mathrm{~g}, 95 \%)$. IR (KBr) $\mathrm{cm}^{-1}$ : 3235. ${ }^{1} \mathrm{H}-\mathrm{NMR}\left(\mathrm{CDCl}_{3}\right) \delta: 2.00(1 \mathrm{H}, \mathrm{m}), 2.10(1 \mathrm{H}$, m), $2.80(1 \mathrm{H}, \mathrm{s}), 3.40(1 \mathrm{H}, \mathrm{m}), 3.60(1 \mathrm{H}, \mathrm{m}), 7.30(5 \mathrm{H}, \mathrm{m})$. ${ }^{13} \mathrm{C}-\mathrm{NMR}\left(\mathrm{CDCl}_{3}\right) \delta: 41.28,41.64,71.04,125.55,127.58$, $128.34,143.32$

3-Iodo-1-phenylpropan-1-ol (4) To a solution of 3chloro-1-phenyl-1-propanol $3(10.0 \mathrm{~g}, 58.6 \mathrm{mmol})$ in acetone $(250 \mathrm{ml})$ was added sodium iodide $(14.9 \mathrm{~g}, 99.6 \mathrm{mmol})$. The mixture was heated to reflux temperature for $48 \mathrm{~h}$. After the solution was cooled to room temperature, the solvent was evaporated in vacuo. The residue was extracted with diethyl ether, the organic layer was washed with brine, dried over $\mathrm{Na}_{2} \mathrm{SO}_{4}$ and evaporated in vacuo to provide the title compound as a yellow solid $(15 \mathrm{~g}, 97 \%)$. mp: $33^{\circ} \mathrm{C}$. IR $(\mathrm{KBr})$ $\mathrm{cm}^{-1}: 3251 .{ }^{1} \mathrm{H}-\mathrm{NMR}\left(\mathrm{CDCl}_{3}\right) \delta: 2.10(2 \mathrm{H}, \mathrm{m}), 2.54(1 \mathrm{H}, \mathrm{s})$, $3.11(1 \mathrm{H}, \mathrm{m}), 3.23(1 \mathrm{H}, \mathrm{m}), 4.71(1 \mathrm{H}, \mathrm{m}), 7.29(5 \mathrm{H}, \mathrm{m}) .{ }^{13} \mathrm{C}-$ NMR $\left(\mathrm{CDCl}_{3}\right) \delta: 2.97,42.15,73.93,125.61,127.68,128.40$, 143.09

1-Phenyl-3-(pyrrolidin-1-yl)propan-1-ol (5a) To a solution of pyrrolidine $(1.59 \mathrm{ml}, 19.1 \mathrm{mmol})$ in tetrahydrofuran (THF) $(7.5 \mathrm{ml})$ was added 3-iodo-1-phenylpropan-1-ol 4 $(1.00 \mathrm{~g}, 3.82 \mathrm{mmol})$ in THF $(2 \mathrm{ml})$ dropwise. The resulting solution was heated to reflux temperature for $4 \mathrm{~h}$. After being cooled to room temperature, the mixture was extracted with dichloromethane and the organic layer was washed with brine, dried over $\mathrm{Na}_{2} \mathrm{SO}_{4}$, and concentrated in vacuo. The crude product was purified by silica gel column chromatography (dichloromethane/methanol, 15/1) to provide the title compound as a pale yellow solid $(450 \mathrm{mg}, 57 \%)$. mp: $72^{\circ} \mathrm{C}$. IR $(\mathrm{KBr}) \mathrm{cm}^{-1}$ : 3111. ${ }^{1} \mathrm{H}-\mathrm{NMR}\left(\mathrm{CDCl}_{3}\right) \delta: 1.79(4 \mathrm{H}, \mathrm{m})$, $1.87(2 \mathrm{H}, \mathrm{m}), 2.53(2 \mathrm{H}, \mathrm{m}), 2.62(3 \mathrm{H}, \mathrm{m}), 2.89(1 \mathrm{H}, \mathrm{m}), 4.95$ $(1 \mathrm{H}, \mathrm{m}), 7.21(1 \mathrm{H}, \mathrm{m}), 7.34(4 \mathrm{H}, \mathrm{m}) .{ }^{13} \mathrm{C}-\mathrm{NMR}\left(\mathrm{CDCl}_{3}\right) \delta$ : $23.48,35.74,54.03,54.78,75.73,125.36,126.60,127.93$, 144.94.

3-(1H-Imidazol-1-yl)-1-phenylpropan-1-ol (5b) Imidazole $(1.30 \mathrm{~g}, 19.1 \mathrm{mmol})$ and 3-iodo-1-phenylpropan-1-ol 4 $(1.00 \mathrm{~g}, 3.82 \mathrm{mmol})$ were subjected to the same reaction described for the synthesis of the compound 5a to give the title compound as a pale yellow solid $(350 \mathrm{mg}, 45 \%)$. mp: $102^{\circ} \mathrm{C}$. IR $(\mathrm{KBr}) \mathrm{cm}^{-1}: 3169 .{ }^{1} \mathrm{H}-\mathrm{NMR}\left(\mathrm{CDCl}_{3}\right) \delta: 1.98(2 \mathrm{H}, \mathrm{m})$, $3.83(1 \mathrm{H}, \mathrm{m}), 4.02(1 \mathrm{H}, \mathrm{m}), 4.37(1 \mathrm{H}, \mathrm{m}), 6.73(2 \mathrm{H}, \mathrm{d}$, $J=7.2 \mathrm{~Hz}), 7.17(6 \mathrm{H}, \mathrm{m}) .{ }^{13} \mathrm{C}-\mathrm{NMR}\left(\mathrm{CDCl}_{3}\right) \delta: 39.96,43.65$, $69.54,118.61,125.45,127.12,128.13,128.39,136.83$, 144.40.

1-Phenyl-3-(piperidin-1-yl)propan-1-ol (5c) Piperidine $(1.89 \mathrm{ml}, 19.2 \mathrm{mmol})$ and 3-iodo-1-phenylpropan-1-ol 4 $(1.00 \mathrm{~g}, 3.82 \mathrm{mmol})$ were subjected to the same reaction described for the synthesis of the compound $\mathbf{5 a}$ to give the title compound as a white solid $(480 \mathrm{mg}, 57 \%)$. mp: $59^{\circ} \mathrm{C}$. IR $(\mathrm{KBr}) \mathrm{cm}^{-1}: 3108 .{ }^{1} \mathrm{H}-\mathrm{NMR}\left(\mathrm{CDCl}_{3}\right) \delta: 1.37(2 \mathrm{H}, \mathrm{s}), 1.55$ $(4 \mathrm{H}, \mathrm{m}), 1.75(2 \mathrm{H}, \mathrm{m}), 2.34(2 \mathrm{H}, \mathrm{s}), 2.45(2 \mathrm{H}, \mathrm{m}), 2.55(2 \mathrm{H}$, $\mathrm{m}), 4.80(1 \mathrm{H}, \mathrm{t}, J=5.2 \mathrm{~Hz}), 6.84(1 \mathrm{H}, \mathrm{s}), 7.11(1 \mathrm{H}, \mathrm{t}$, $J=6.4 \mathrm{~Hz}), 7.23(4 \mathrm{H}, \mathrm{m}) .{ }^{13} \mathrm{C}-\mathrm{NMR}\left(\mathrm{CDCl}_{3}\right) \delta: 23.95,25.70$,
33.50, 54.30, 57.33, 74.99, 125.18, 126.44, 127.78, 144.66

3-Morpholino-1-phenylpropan-1-ol (5d) Morpholine $(1.67 \mathrm{ml}, 19.1 \mathrm{mmol})$ and 3-iodo-1-phenylpropan-1-ol 4 $(1.00 \mathrm{~g}, 3.82 \mathrm{mmol})$ were subjected to the same reaction described for the synthesis of the compound 5a to give the title compound as a pale yellow solid $(450 \mathrm{mg}, 53 \%)$. mp: $60^{\circ} \mathrm{C}$. IR $(\mathrm{KBr}) \mathrm{cm}^{-1}: 3162 .{ }^{1} \mathrm{H}-\mathrm{NMR}\left(\mathrm{CDCl}_{3}\right) \delta: 1.85(2 \mathrm{H}, \mathrm{m})$, $2.47(2 \mathrm{H}, \mathrm{s}), 2.60(4 \mathrm{H}, \mathrm{m}), 3.72(4 \mathrm{H}, \mathrm{t}, J=4.8 \mathrm{~Hz}), 4.90(1 \mathrm{H}$, $\mathrm{t}, J=5.6 \mathrm{~Hz}), 7.22(1 \mathrm{H}, \mathrm{m}), 7.32(4 \mathrm{H}, \mathrm{m}) .{ }^{13} \mathrm{C}-\mathrm{NMR}\left(\mathrm{CDCl}_{3}\right)$ $\delta: 33.41,53.52,57.33,66.77,75.19,125.22,126.71,127.96$, 144.41. MS $m / z: 222.00\left([\mathrm{M}+\mathrm{H}]^{+}\right)$.

3-(4-Methylpiperazin-1-yl)-1-phenylpropan-1-ol (5e) 1-Methylpiperazine $(2.12 \mathrm{ml}, 19.1 \mathrm{mmol})$ and 3-iodo-1phenylpropan-1-ol $4(1.00 \mathrm{~g}, 3.82 \mathrm{mmol})$ were subjected to the same reaction described for the synthesis of the compound $\mathbf{5 a}$ to give the title compound as a white solid (450 mg, 50\%). mp: $71^{\circ} \mathrm{C}$. IR (KBr) cm $\mathrm{cm}^{-1}$ : 3114, 2948, 2806. ${ }^{1} \mathrm{H}-\mathrm{NMR}\left(\mathrm{CDCl}_{3}\right) \delta: 1.71(2 \mathrm{H}, \mathrm{m}), 2.11(3 \mathrm{H}, \mathrm{s}), 2.33$ $(6 \mathrm{H}, \mathrm{m}), 2.48(4 \mathrm{H}, \mathrm{m}), 4.70(1 \mathrm{H}, \mathrm{t}, J=5.6 \mathrm{~Hz}), 7.08(1 \mathrm{H}, \mathrm{m})$, $7.20(4 \mathrm{H}, \mathrm{m}) .{ }^{13} \mathrm{C}-\mathrm{NMR}\left(\mathrm{CDCl}_{3}\right) \delta: 33.79,45.27,52.30$, $54.35,55.89,73.91,124.88,126.18,127.43,144.40$

tert-Butyl 4-(3-Hydroxy-3-phenylpropyl)piperazine-1carboxylate (5f) Piperazine $(5.92 \mathrm{~g}, 68.7 \mathrm{mmol})$ and 3 iodo-1-phenylpropan-1-one $4(3.00 \mathrm{~g}, 11.4 \mathrm{mmol})$ were subjected to the same reaction described for the synthesis of the compound 5a. To a solution of crude product in THF $(10 \mathrm{ml})$ was added sodium bicarbonate $(3.81 \mathrm{~g}, 45.4 \mathrm{mmol})$ and ditert-butyl dicarbonate $(2.38 \mathrm{~g}, 10.9 \mathrm{mmol})$ in $\mathrm{H}_{2} \mathrm{O} / \mathrm{THF}(1 / 1$, $60 \mathrm{ml}$ ). The solution was stirred for $1 \mathrm{~h}$ at room temperature. The mixture was extracted with ethyl acetate, washed with brine, dried over $\mathrm{Na}_{2} \mathrm{SO}_{4}$, and concentrated in vacuo. The crude product was purified by silica gel column chromatography $(\mathrm{EA} / \mathrm{HX}, 5 / 1)$ to give the title compound as a colorless oil $(1 \mathrm{~g}, 27 \%)$. IR (KBr) $\mathrm{cm}^{-1}: 3120,1686 .{ }^{1} \mathrm{H}-\mathrm{NMR}$ $\left(\mathrm{CDCl}_{3}\right) \delta: 1.46(9 \mathrm{H}, \mathrm{s}), 1.86(2 \mathrm{H}, \mathrm{m}), 2.43(2 \mathrm{H}, \mathrm{m}), 2.58$ $(4 \mathrm{H}, \mathrm{m}), 2.66(1 \mathrm{H}, \mathrm{m}), 3.47(4 \mathrm{H}, \mathrm{t}, J=4.8 \mathrm{~Hz}), 4.92(1 \mathrm{H}, \mathrm{t}$, $J=6.0 \mathrm{~Hz}), 7.24(1 \mathrm{H}, \mathrm{m}), 7.33(4 \mathrm{H}, \mathrm{m}) .{ }^{13} \mathrm{C}-\mathrm{NMR}\left(\mathrm{CDCl}_{3}\right)$ $\delta: 28.47,33.74,53.06,57.12,75.41,79.79,125.33,126.83$, $128.09,144.46,154.37$.

1-(3-Phenyl-3-(4-(trifluoromethyl)phenoxy)propyl)pyrrolidine (6a) To a solution of $\mathrm{NaH}$ (250 mg, $10.0 \mathrm{mmol})$ in $N, N$-dimethylethanamide (DMA) $(10 \mathrm{ml})$ was added 1-phenyl-3-(pyrrolidin-1-yl)propan-1-ol 5a (410 mg, $2.00 \mathrm{mmol}$ ). The mixture was stirred at $70^{\circ} \mathrm{C}$ for $30 \mathrm{~min} . p$ Chlorobenzotrifluoride $(1.34 \mathrm{ml}, 10.0 \mathrm{mmol})$ was added and the mixture was heated to $100^{\circ} \mathrm{C}$ for $2 \mathrm{~h}$. After being cooled to room temperature, the mixture was quenched with $1 \mathrm{~N}$ $\mathrm{NaOH}$ solution $(10 \mathrm{ml})$ and extracted with toluene. The organic layer was washed with brine, dried over $\mathrm{Na}_{2} \mathrm{SO}_{4}$ and concentrated in vacuo. The crude product was purified by silica gel column chromatography $(\mathrm{EA} / \mathrm{HX}, 1 / 1)$ to give the title compound as a pale yellow oil (200 mg, 29\%). ${ }^{1} \mathrm{H}-\mathrm{NMR}$ $\left(\mathrm{CDCl}_{3}\right) \delta: 1.43(2 \mathrm{H}, \mathrm{m}), 1.60(4 \mathrm{H}, \mathrm{m}), 2.05(1 \mathrm{H}, \mathrm{m}), 2.22$ $(1 \mathrm{H}, \mathrm{m}), 2.43(4 \mathrm{H}, \mathrm{s}), 5.25(1 \mathrm{H}, \mathrm{q}, J=4.8 \mathrm{~Hz}), 6.88(2 \mathrm{H}, \mathrm{d}$, $J=8.8 \mathrm{~Hz}), 7.22(1 \mathrm{H}, \mathrm{m}), 7.30(4 \mathrm{H}, \mathrm{m}), 7.38(2 \mathrm{H}, \mathrm{d}$, $J=8.8 \mathrm{~Hz}) .{ }^{13} \mathrm{C}-\mathrm{NMR}\left(\mathrm{CDCl}_{3}\right) \delta: 23.52,34.43,52.43,35.66$, $54.40,115.60,122.64 \quad\left(J_{\mathrm{C}-\mathrm{F}}=31.9 \mathrm{~Hz}\right), 125.55,126.65$, $126.68,128.27,128.87,138.66,159.36$. MS $\mathrm{m} / \mathrm{z}: 350.15$ $\left([\mathrm{M}+\mathrm{H}]^{+}\right)$.

1-(3-Phenyl-3-(4-(trifluoromethyl)phenoxy)propyl)-1Himidazole (6b) Sodium hydride (178 mg, $7.42 \mathrm{mmol}), 3-$ 
(1H-imidazol-1-yl)-1-phenylpropan-1-ol 5b (300 mg, 1.48 mmol) and $p$-chlorobenzotrifluoride $(1.00 \mathrm{ml}, 7.42 \mathrm{mmol})$ were subjected to the same reaction described for the synthesis of the compound $\mathbf{6 a}$ to give the title compound as a pale yellow oil $(140 \mathrm{mg}, 27 \%) .{ }^{1} \mathrm{H}-\mathrm{NMR}\left(\mathrm{CDCl}_{3}\right) \delta: 2.28(1 \mathrm{H}$, $\mathrm{m}), 2.45(1 \mathrm{H}, \mathrm{m}), 4.10(1 \mathrm{H}, \mathrm{m}), 4.24(1 \mathrm{H}, \mathrm{m}), 5.00(1 \mathrm{H}, \mathrm{q}$, $J=3.6 \mathrm{~Hz}), 6.85(2 \mathrm{H}, \mathrm{t}, J=8.8 \mathrm{~Hz}), 7.06(1 \mathrm{H}, \mathrm{s}), 7.26(2 \mathrm{H}$, $\mathrm{m}), 7.32(2 \mathrm{H}, \mathrm{m}), 7.43(2 \mathrm{H}, \mathrm{m}), 7.50(1 \mathrm{H}, \mathrm{d}, J=8.4 \mathrm{~Hz}), 7.75$ $(1 \mathrm{H}, \mathrm{d}, J=8.8 \mathrm{~Hz}), 7.90(1 \mathrm{H}, \mathrm{s}) .{ }^{13} \mathrm{C}-\mathrm{NMR}\left(\mathrm{CDCl}_{3}\right) \delta: 39.82$, 43.18, 76.37, 115.36, 119.20, $122.56 \quad\left(J_{\mathrm{C}-\mathrm{F}}=23.7 \mathrm{~Hz}\right)$, $125.33,126.59,126.62,128.00,128.20,128.72,137.30$, 139.28, 159.49. MS $m / z: 347.15\left([\mathrm{M}+\mathrm{H}]^{+}\right)$.

1-(3-Phenyl-3-(4-(trifluoromethyl)phenoxy)propyl)piperidine (6c) Sodium hydride (246 mg, $10.3 \mathrm{mmol}), 1$ phenyl-3-(piperidin-1-yl)propan-1-ol 5c (450 mg, $2.05 \mathrm{mmol})$ and $p$-chlorobenzotrifluoride $(1.38 \mathrm{ml}, 10.3 \mathrm{mmol})$ were subjected to the same reaction described for the synthesis of the compound $\mathbf{6 a}$ to give the title compound as a pale red oil $(150 \mathrm{mg}, 20 \%) .{ }^{1} \mathrm{H}-\mathrm{NMR}\left(\mathrm{CDCl}_{3}\right) \delta: 1.25(2 \mathrm{H}, \mathrm{s}), 1.41(4 \mathrm{H}$, m), $1.85(1 \mathrm{H}, \mathrm{m}), 2.07(1 \mathrm{H}, \mathrm{m}), 2.20(4 \mathrm{H}, \mathrm{s}), 2.28(2 \mathrm{H}, \mathrm{m})$, $5.13(1 \mathrm{H}, \mathrm{t}, J=8.0 \mathrm{~Hz}), 6.74(2 \mathrm{H}, \mathrm{d}, J=8.4 \mathrm{~Hz}), 7.03(1 \mathrm{H}$, $\mathrm{m}), 7.11(2 \mathrm{H}, \mathrm{t}, J=8.0 \mathrm{~Hz}), 7.20(4 \mathrm{H}, \mathrm{m}) .{ }^{13} \mathrm{C}-\mathrm{NMR}\left(\mathrm{CDCl}_{3}\right)$ $\delta: 24.29,25.88,35.86,54.35,54.92,78.59,115.44,122.06$ $\left(J_{\mathrm{C}-\mathrm{F}}=32.6 \mathrm{~Hz}\right), 125.51,126.21,126.28,127.33,128.27$, 140.83, 160.36. MS m/z: $363.95\left([\mathrm{M}+\mathrm{H}]^{+}\right), \quad 379.95$ $\left(\left[\mathrm{M}+\mathrm{NH}_{4}\right]^{+}\right)$.

4-(3-Phenyl-3-(4-(trifluoromethyl)phenoxy)propyl)morpholine (6d) Sodium hydride (217 mg, $9.04 \mathrm{mmol}), 3$-morpholino-1-phenylpropan-1-ol 5d (400 mg, $1.81 \mathrm{mmol})$ and $p$ chlorobenzotriffuoride $(1.22 \mathrm{ml}, 9.04 \mathrm{mmol})$ were subjected to the same reaction described for the synthesis of the compound 6a to give the title compound as a pale yellow oil $(150 \mathrm{mg}, 23 \%) .{ }^{1} \mathrm{H}-\mathrm{NMR}\left(\mathrm{CDCl}_{3}\right) \delta: 1.90(1 \mathrm{H}, \mathrm{m}), 2.13(1 \mathrm{H}$, m), $2.36(6 \mathrm{H}, \mathrm{m}), 3.59(4 \mathrm{H}, \mathrm{t}, J=4.4 \mathrm{~Hz}), 5.18(1 \mathrm{H}, \mathrm{q}$, $J=5.2 \mathrm{~Hz}), 6.78(2 \mathrm{H}, \mathrm{d}, J=8.4 \mathrm{~Hz}), 7.12(1 \mathrm{H}, \mathrm{m}), 7.20(4 \mathrm{H}$, m), $7.34(2 \mathrm{H}, \mathrm{d}, J=8.8 \mathrm{~Hz}) .{ }^{13} \mathrm{C}-\mathrm{NMR}\left(\mathrm{CDCl}_{3}\right) \delta: 35.66$, $53.70,54.84,66.94,78.44,115.58,122.38\left(J_{\mathrm{C}-\mathrm{F}}=32.6 \mathrm{~Hz}\right)$, $125.67,126.52,126.56,127.68,128.57,140.76,160.36 . \mathrm{MS}$ $m / z: 365.95\left([\mathrm{M}+\mathrm{H}]^{+}\right)$.

1-Methyl-4-(3-phenyl-3-(4-(trifluoromethyl)phenoxy)propyl)piperazine (6e) Sodium hydride (205 mg, 8.53 mmol), 3-(4-methylpiperazin-1-yl)-1-phenylpropan-1-ol 5e $(400 \mathrm{mg}, 1.71 \mathrm{mmol})$ and $p$-chlorobenzotrifluoride $(1.15 \mathrm{ml}$, $8.53 \mathrm{mmol}$ ) were subjected to the same reaction described for the synthesis of the compound $\mathbf{6 a}$ to give the title compound as a pale yellow oil $(100 \mathrm{mg}, 15 \%) .{ }^{1} \mathrm{H}-\mathrm{NMR}\left(\mathrm{CDCl}_{3}\right) \delta$ : $1.85(1 \mathrm{H}, \mathrm{m}), 2.08(1 \mathrm{H}, \mathrm{m}), 2.16(3 \mathrm{H}, \mathrm{s}), 2.32(10 \mathrm{H}, \mathrm{m}), 5.15$ $(1 \mathrm{H}, \mathrm{m}), 6.77(2 \mathrm{H}, \mathrm{d}, J=8.8 \mathrm{~Hz}), 7.09(1 \mathrm{H}, \mathrm{m}), 7.18(4 \mathrm{H}, \mathrm{m})$, $7.27(2 \mathrm{H}, \mathrm{d}, J=8.4 \mathrm{~Hz}) \cdot{ }^{13} \mathrm{C}-\mathrm{NMR}\left(\mathrm{CDCl}_{3}\right) \delta: 35.88,45.85$, $52.98,54.17,55.00,78.40,115.44,125.42,122.13\left(J_{\mathrm{C}-}\right.$ $\mathrm{F}=32.6 \mathrm{~Hz}), 126.33,126.36,127.45,128.36,140.70,160.27$. MS $m / z: 379.20\left([\mathrm{M}+\mathrm{H}]^{+}\right)$.

1-(3-Phenyl-3-(4-(trifluoromethyl)phenoxy)propyl)piperazine (6f) Sodium hydride $(374 \mathrm{mg}, 15.6 \mathrm{mmol})$, tertbutyl 4-(3-hydroxy-3-phenylpropyl)piperazine-1-carboxylate 5f $(1.00 \mathrm{~g}, 3.12 \mathrm{mmol})$ and $p$-chlorobenzotrifluoride $(2.10 \mathrm{ml}$, $15.6 \mathrm{mmol}$ ) were subjected to the same reaction described for the synthesis of the compound $\mathbf{6 a}$ to give crude yellow oil. The crude oil was dissolved in 1,4-dioxane $(5 \mathrm{ml})$ and $4 \mathrm{~N}$ $\mathrm{HCl}$ in 1,4-dioxane $(30 \mathrm{ml})$ added. This solution was stirred for $3 \mathrm{~h}$ at room temperature. After the solution was evapo- rated in vacuo, the residue was dissolved in ethyl acetate and then basified with $1 \mathrm{~N} \mathrm{NaOH}$ solution. The organic layer was washed with brine, dried over $\mathrm{Na}_{2} \mathrm{SO}_{4}$ and concentrated in vacuo to give the title compound as a yellow oil $(500 \mathrm{mg}$, 44\%). ${ }^{1} \mathrm{H}-\mathrm{NMR}\left(\mathrm{CDCl}_{3}\right) \delta: 1.84(1 \mathrm{H}, \mathrm{s}), 2.00(1 \mathrm{H}, \mathrm{m}), 2.20$ $(1 \mathrm{H}, \mathrm{m}), 2.45(6 \mathrm{H}, \mathrm{m}), 2.90(4 \mathrm{H}, \mathrm{m}), 5.27(1 \mathrm{H}, \mathrm{q}, J=5.2 \mathrm{~Hz})$, $6.89(2 \mathrm{H}, \mathrm{d}, J=8.8 \mathrm{~Hz}), 7.23(1 \mathrm{H}, \mathrm{m}), 7.32(4 \mathrm{H}, \mathrm{m}), 7.41$ $(2 \mathrm{H}, \mathrm{d}, J=8.4 \mathrm{~Hz}) .{ }^{13} \mathrm{C}-\mathrm{NMR}\left(\mathrm{CDCl}_{3}\right) \delta: 35.75,46.05,54.46$, $54.98,78.48, \quad 115.49,122.18 \quad\left(J_{\mathrm{C}-\mathrm{F}}=31.8 \mathrm{~Hz}\right), 122.48$, $126.42,126.46,127.53,128.45,140.77,160.30$. MS $\mathrm{m} / \mathrm{z}$ : $364.90\left([\mathrm{M}+\mathrm{H}]^{+}\right)$.

3-Iodo-1-phenylpropan-1-one (9a) To a solution of 3chloro-1-propiophenone $2(5.00 \mathrm{~g}, 29.7 \mathrm{mmol})$ in acetone $(100 \mathrm{ml})$ was added sodium iodide $(7.56 \mathrm{~g}, 50.4 \mathrm{mmol})$. The mixture was heated to reflux temperature for $4 \mathrm{~h}$. After the solution was cooled to room temperature, the solvent was evaporated in vacuo. The residue was extracted with diethyl ether, the organic layer was washed with brine, dried over $\mathrm{Na}_{2} \mathrm{SO}_{4}$ and evaporated in vacuo to provide the title compound as a yellow solid $(4.14 \mathrm{~g}, 54 \%)$. mp: $56^{\circ} \mathrm{C}$. IR $(\mathrm{KBr})$ $\mathrm{cm}^{-1}: 1675 .{ }^{1} \mathrm{H}-\mathrm{NMR}\left(\mathrm{CDCl}_{3}\right) \delta: 3.32(2 \mathrm{H}, \mathrm{t}, J=6.4 \mathrm{~Hz})$, $3.48(2 \mathrm{H}, \mathrm{t}, J=7.6 \mathrm{~Hz}), 7.34(2 \mathrm{H}, \mathrm{m}), 7.44(1 \mathrm{H}, \mathrm{m}), 7.79$ $(2 \mathrm{H}, \mathrm{m}) .{ }^{13} \mathrm{C}-\mathrm{NMR}\left(\mathrm{CDCl}_{3}\right) \delta: 3.58,42.31,127.62,128.36$, 133.13, 135.63, 196.90.

2-Bromo-1-phenylethanone (9b) To a solution of triphenylphosphine $(2.89 \mathrm{~g}, 11.1 \mathrm{mmol})$ and carbon tetrabromide $(3.69 \mathrm{~g}, 11.1 \mathrm{mmol})$ in acetonitrile $(10 \mathrm{ml})$ and dichloromethane $(15 \mathrm{ml})$ at $0{ }^{\circ} \mathrm{C}$ was added 2-hydroxyacetophenone 7 (500 mg, $3.67 \mathrm{mmol})$ in acetonitrile $(5 \mathrm{ml})$. After being stirred for $15 \mathrm{~min}$, the mixture was filtered off and the residue was concentrated in vacuo. The crude product was purified by silica column chromatography $(\mathrm{EA} / \mathrm{HX}, 1 / 3)$ to give the title compound as a yellow oil $(200 \mathrm{mg}, 73 \%)$. IR $(\mathrm{KBr}) \mathrm{cm}^{-1}: 1680 .{ }^{1} \mathrm{H}-\mathrm{NMR}\left(\mathrm{CDCl}_{3}\right) \delta: 4.37(2 \mathrm{H}, \mathrm{s}), 7.39$ $(2 \mathrm{H}, \mathrm{t}, J=7.6 \mathrm{~Hz}), 7.51(1 \mathrm{H}, \mathrm{t}, J=7.6 \mathrm{~Hz}), 7.87(2 \mathrm{H}, \mathrm{m}) .{ }^{13} \mathrm{C}-$ NMR $\left(\mathrm{CDCl}_{3}\right) \delta: 31.06,128.68,128.72,133.76,190.93$.

4-Iodo-1-phenylbutan-1-one (9c) To a solution of 4chloro-1-phenylbutan-1-one $8(1.00 \mathrm{~g}, 5.47 \mathrm{mmol})$ in acetone $(30 \mathrm{ml})$ was added sodium iodide $(1.22 \mathrm{~g}, 8.21 \mathrm{mmol})$. The mixture was heated to reflux temperature for $18 \mathrm{~h}$. After the solution was cooled to room temperature, the solvent was evaporated in vacuo. The residue was extracted with diethyl ether, the organic layer was washed with brine, dried over $\mathrm{Na}_{2} \mathrm{SO}_{4}$ and evaporated in vacuo to provide the title compound as a yellow solid $(800 \mathrm{mg}, 80 \%)$. IR $(\mathrm{KBr}) \mathrm{cm}^{-1}$ : 1693. ${ }^{1} \mathrm{H}-\mathrm{NMR}\left(\mathrm{CDCl}_{3}\right) \delta: 2.19(2 \mathrm{H}, \mathrm{q}, J=6.4 \mathrm{~Hz}), 3.06$ $(2 \mathrm{H}, \mathrm{t}, J=6.8 \mathrm{~Hz}), 3.25(2 \mathrm{H}, \mathrm{t}, J=6.8 \mathrm{~Hz}), 7.38(2 \mathrm{H}, \mathrm{m}), 7.48$ $(1 \mathrm{H}, \mathrm{m}), 7.88(2 \mathrm{H}, \mathrm{m}) .{ }^{13} \mathrm{C}-\mathrm{NMR}\left(\mathrm{CDCl}_{3}\right) \delta: 6.97,27.61$, $38.99,127.87,128.51,133.08,136.53,198.29$.

3-Morpholino-1-phenylpropan-1-one (10a) Morpholine $(8.40 \mathrm{ml}, 96.0 \mathrm{mmol})$ and 3-iodo-1-phenylpropan-1-one 9a $(500 \mathrm{mg}, 1.92 \mathrm{mmol})$ were subjected to the same reaction described for the synthesis of the compound $\mathbf{5 a}$ to give the title compound as a yellow oil $(350 \mathrm{mg}, 83 \%)$. The title compound was previously synthesized from dimethyaminopropiophenone as a hydrochloride salt $\left(\mathrm{mp}^{23)}=175-177^{\circ} \mathrm{C}\right)$. IR (KBr) $\mathrm{cm}^{-1}: 1685 .{ }^{1} \mathrm{H}-\mathrm{NMR}\left(\mathrm{CDCl}_{3}\right) \delta: 2.43(4 \mathrm{H}, \mathrm{t}, J=$ $4.4 \mathrm{~Hz}), 2.75(2 \mathrm{H}, \mathrm{t}, J=7.2 \mathrm{~Hz}), 3.10(2 \mathrm{H}, \mathrm{t}, J=7.6 \mathrm{~Hz}), 3.63$ $(4 \mathrm{H}, \mathrm{t}, J=4.8 \mathrm{~Hz}), 7.37(2 \mathrm{H}, \mathrm{t}, J=8.0 \mathrm{~Hz}), 7.47(1 \mathrm{H}, \mathrm{t}$, $J=7.2 \mathrm{~Hz}), 7.86(2 \mathrm{H}, \mathrm{m}) .{ }^{13} \mathrm{C}-\mathrm{NMR}\left(\mathrm{CDCl}_{3}\right) \delta: 35.98,53.51$, $53.66,66.84,127.81,128.44,132.95,136.63,198.57 . \mathrm{MS}$ 
$m / z: 220.00\left([\mathrm{M}+\mathrm{H}]^{+}\right)$.

2-Morpholino-1-phenylethanone (10b) Morpholine (439 $\mu \mathrm{l}, 5.00 \mathrm{mmol})$ and 2-bromo-1-phenylethanone 9b (200 $\mathrm{mg}, 1.00 \mathrm{mmol})$ were subjected to the same reaction described for the synthesis of the compound $\mathbf{5 a}$ to give the title compound as a yellow oil $(500 \mathrm{mg}, 25 \%)$. IR $(\mathrm{KBr}) \mathrm{cm}^{-1}$ : 1680. ${ }^{1} \mathrm{H}-\mathrm{NMR}\left(\mathrm{CDCl}_{3}\right) \delta: 2.62(4 \mathrm{H}, \mathrm{m}), 3.78(4 \mathrm{H}, \mathrm{m}), 3.83$ $(2 \mathrm{H}, \mathrm{s}), 7.45(2 \mathrm{H}, \mathrm{m}), 7.56(1 \mathrm{H}, \mathrm{m}), 7.97(2 \mathrm{H}, \mathrm{m}) .{ }^{13} \mathrm{C}-\mathrm{NMR}$ $\left(\mathrm{CDCl}_{3}\right) \delta: 53.88,64.64,66.78,127.90,128.44,133.19 . \mathrm{MS}$ $m / z: 206.00\left([\mathrm{M}+\mathrm{H}]^{+}\right)$

4-Morpholino-1-phenylbutan-1-one (10c) Morpholine $(1.12 \mathrm{ml}, 12.8 \mathrm{mmol})$ and 4-iodo-1-phenylbutan-1-one 9c $(0.70 \mathrm{~g}, 2.55 \mathrm{mmol})$ were subjected to the same reaction described for the synthesis of the compound $\mathbf{5 a}$ to give the title compound as a yellow oil $(450 \mathrm{mg}, 40 \%)$. IR $(\mathrm{KBr}) \mathrm{cm}^{-1}$ : 1686. ${ }^{1} \mathrm{H}-\mathrm{NMR}\left(\mathrm{CDCl}_{3}\right) \delta: 1.94(2 \mathrm{H}, \mathrm{q}, J=6.8 \mathrm{~Hz}), 2.40$ $(6 \mathrm{H}, \mathrm{m}), 3.00(2 \mathrm{H}, \mathrm{t}, J=7.2 \mathrm{~Hz}), 3.63(4 \mathrm{H}, \mathrm{t}, J=4.4 \mathrm{~Hz}), 7.43$ $(2 \mathrm{H}, \mathrm{m}), 7.53(1 \mathrm{H}, \mathrm{m}), 7.94(2 \mathrm{H}, \mathrm{m}) .{ }^{13} \mathrm{C}-\mathrm{NMR}\left(\mathrm{CDCl}_{3}\right) \delta$ : $21.21,36.02,53.46,57.99,66.73,127.73,128.22,132.56$, 136.83, 199.43. MS $m / z: 234.00\left([\mathrm{M}+\mathrm{H}]^{+}\right)$.

3-Morpholino-1-phenylpropan-1-one oxime (11a) To a solution of 3-morpholino-1-phenylpropan-1-one 10a (350 $\mathrm{mg}, 1.60 \mathrm{mmol})$ in ethanol $(10 \mathrm{ml})$ was added hydroxylamine hydrochloride $(155 \mathrm{mg}, 2.23 \mathrm{mmol})$ and pyridine $(180 \mu \mathrm{l}$, $2.23 \mathrm{mmol}$ ). The solution was heated to reflux temperature for $1 \mathrm{~h}$. After being cooled to room temperature, the mixture was extracted with ethyl acetate. The organic layer was washed with brine, dried over $\mathrm{Na}_{2} \mathrm{SO}_{4}$ and concentrated in vacuo. The residue was purified by silica gel column chromatography (dichloromethane/methanol, 20/1) to give the title compound as a white powder $(30 \mathrm{mg}, 10 \%)$. mp: $143{ }^{\circ} \mathrm{C}$. IR $(\mathrm{KBr}) \mathrm{cm}^{-1}: 2972,2845 .{ }^{1} \mathrm{H}-\mathrm{NMR} \delta: 2.57(4 \mathrm{H}, \mathrm{s}), 2.63$ $(2 \mathrm{H}, \mathrm{t}, J=8.8 \mathrm{~Hz}), 3.07(2 \mathrm{H}, \mathrm{t}, J=6.8 \mathrm{~Hz}), 3.75(4 \mathrm{H}, \mathrm{m}), 7.30$ $(3 \mathrm{H}, \mathrm{m}), 7.61(2 \mathrm{H}, \mathrm{t}, J=4.4 \mathrm{~Hz}) .{ }^{13} \mathrm{C}-\mathrm{NMR} \delta: 23.94,53.40$, $54.88,66.72,126.02,128.40,128.97,135.60,157.04 . \mathrm{MS}$ $\mathrm{m} / \mathrm{z}: 235.00\left([\mathrm{M}+\mathrm{H}]^{+}\right)$

3-Morpholino-1-phenylpropan-1-one $O$-ethyl oxime (11b) 3-Morpholino-1-phenylpropan-1-one 10a (500 mg, $2.28 \mathrm{mmol}), O$-ethylhydroxylamine hydrochloride $(267 \mathrm{mg}$, $2.74 \mathrm{mmol})$ and pyridine $(221 \mu \mathrm{l}, 2.74 \mathrm{mmol})$ were subjected to the same reaction described for the synthesis of the compound 11a to give the title compound as a colorless oil $(50 \mathrm{mg}, 8 \%) .{ }^{1} \mathrm{H}-\mathrm{NMR}\left(\mathrm{CDCl}_{3}\right) \delta: 1.31(3 \mathrm{H}, \mathrm{t}, J=7.2 \mathrm{~Hz})$, $2.50(6 \mathrm{H}, \mathrm{m}), 2.96(2 \mathrm{H}, \mathrm{m}), 3.70(4 \mathrm{H}, \mathrm{t}, J=4.8 \mathrm{~Hz}), 4.22$ $(2 \mathrm{H}, \mathrm{q}, J=7.2 \mathrm{~Hz}), 7.34(3 \mathrm{H}, \mathrm{m}), 7.62(2 \mathrm{H}, \mathrm{m}) .{ }^{13} \mathrm{C}-\mathrm{NMR}$ $\left(\mathrm{CDCl}_{3}\right) \delta:$ 21.21, 36.02, 53.46, 57.99, 66.73, 127.73, 128.22, 132.56, 136.83, 199.43. MS $m / z: 263.00\left([\mathrm{M}+\mathrm{H}]^{+}\right)$.

3-Morpholino-1-phenylpropan-1-one $O$-benzyl oxime (11c) 3-Morpholino-1-phenylpropan-1-one 10a (500 mg, $2.28 \mathrm{mmol}), O$-benzylhydroxylamine hydrochloride $(437 \mathrm{mg}$, $2.74 \mathrm{mmol})$ and pyridine $(221 \mu \mathrm{l}, 2.74 \mathrm{mmol})$ were subjected to the same reaction described for the synthesis of 11a to give the title compound as a colorless oil $(100 \mathrm{mg}, 14 \%) .{ }^{1} \mathrm{H}-$ NMR $\left(\mathrm{CDCl}_{3}\right) \delta: 2.42(4 \mathrm{H}, \mathrm{m}), 2.52(2 \mathrm{H}, \mathrm{m}), 2.97(2 \mathrm{H}, \mathrm{m})$, $3.63(4 \mathrm{H}, \mathrm{t}, J=4.4 \mathrm{~Hz}), 5.21(2 \mathrm{H}, \mathrm{s}), 7.27(1 \mathrm{H}, \mathrm{m}), 7.32(5 \mathrm{H}$ m), $7.38(2 \mathrm{H}, \mathrm{m}) 7.60(2 \mathrm{H}, \mathrm{m}) .{ }^{13} \mathrm{C}-\mathrm{NMR}\left(\mathrm{CDCl}_{3}\right) \delta: 24.66$, 53.35, 54.93, 66.82, 126.08, 127.53, 127.91, 128.08, 128.20, 128.86, 125.41, 137.69, 156.77. MS $m / z: 324.95\left([\mathrm{M}+\mathrm{H}]^{+}\right)$.

BV2 Cell Culture BV2 cells were grown in Dulbecco's modified Eagle's medium (DMEM; Gibco, U.S.A.) containing 5\% FBS (Hyclone, U.S.A.) and 1\% penicillin-strepto- mycin (Gibco, U.S.A.). ${ }^{24)}$

Determination of NO Release BV2 cells $\left(2 \times 10^{5}\right)$ were treated with LPS $(100 \mathrm{ng} / \mathrm{ml})$ for $24 \mathrm{~h}$. To measure the amount of NO produced by the BV2 cells, $100 \mu \mathrm{l}$ of conditioned medium was mixed with an equal volume of Griess reagent $(0.5 \%$ sulfanilamide and $0.05 \% \mathrm{~N}$-1-naphthylethylenediamine), and incubated for $10 \mathrm{~min}$ at room temperature. The absorbance of the mixture at $550 \mathrm{~nm}$ was measured using a microplate reader. ${ }^{24)}$

Cell Viability Cell respiration, an indicator of cell viability, was determined by the mitochondrial-dependent reduction of 3-(4,5-dimethylthiazol-2-yl)-2,5-diphenyltetrazoium (MTT; Sigma-Aldrich Corp., U.S.A.) to formazan. MTT solution $(50 \mu \mathrm{l}, 0.5 \mathrm{mg} / \mathrm{ml})$ was added to BV2 cell cultures. The conversion of MTT to formazan by metabolically visible cells was measured with a microplate reader at $595 \mathrm{~nm}^{15)}$

Reverse Transcription-Polymerase Chain Reaction Total RNA was prepared with TriZol reagent (Gibco BRL, U.S.A.), and $1-\mu \mathrm{g}$ RNA samples were used for cDNA synthesis using RT-PCR kit (Roche, Germany). The primer sequences of rat inducible NO synthase (iNOS) was described previously. ${ }^{25)}$

Immunoblotting BV2 cells were washed twice with cold phosphate buffered saline (PBS) and lysed in RIPA buffer (50 mm Tris-HCl, pH 7.4, 1\% NP-40, 0.25\% sodiumdeoxycholate, $150 \mathrm{~mm} \mathrm{NaCl}, 1 \mathrm{~mm} \mathrm{Na} \mathrm{VO}_{4}$, and 1 complete Mini protease inhibitor cocktail tablet (Roche Diagnostics, Switzerland)). The lysate was centrifuged for $15 \mathrm{~min}$ at $12000 \mathrm{rpm}$ at $4{ }^{\circ} \mathrm{C}$ and supernatants were loaded onto $6 \%$ sodium dodecyl sulfate-polyacrylamide gel electrophoresis (SDS-PAGE). Primary antibodies were diluted as follows: $1: 1000$ for anti-iNOS (BD Bioscience, U.S.A.). The antigenantibody complex was detected by BM Chemiluminescence Blotting Substrate (Roche, Germany) using donkey antirabbit horseradish peroxidase-conjugated secondary antibody (Santa Cruz Biotechnology, U.S.A.). ${ }^{25)}$

\section{RESULTS AND DISCUSSION}

Chemistry of Fluoxetine Analogues The syntheses of the heterocyclic analogues of fluoxetine started from commercially available 3 -chloropropiophenone $\mathbf{2}$, as depicted in Chart 1. Since racemic fluoxetine is used therapeutically, all compounds were synthesized as a racemic mixture. Chloropropanone 2 was first reduced to chloropropanol 3 using 3 eq of $\mathrm{NaBH}_{4}$ in methanol at room temperature for $24 \mathrm{~h}$ in excellent yield (95\%). The chloropropanol 3 was converted to iodopropanol 4 using $\mathrm{NaI}$ with acetone via a Finkelstein reaction in $97 \%$ yield and the completion of the reaction was judged by the ${ }^{1} \mathrm{H}-\mathrm{NMR}$ spectrum. ${ }^{26)}$ Reactions of iodopropanol 4 with various heterocyclic amines produced the crude products of corresponding amine-propanols $\mathbf{5 a}-\mathbf{f}$ containing excess amines, which were purified by chromatography on silica gel with dichloromethane/methanol (v/v, 15/1) to give pure amine-propanols $(\mathbf{5 a}-\mathbf{f})$. Finally, the heterocyclic analogues $\mathbf{6 a}-\mathbf{f}$ were prepared by nucleophilic aromatic substitution of $p$-chlorobenzotrifluoride with the corresponding alkoxide anions of compounds $(\mathbf{5 a}-\mathbf{f})$, which were generated by reacting with sodium hydride in DMA.

The simplified analogues of fluoxetine, where the trifluo- 


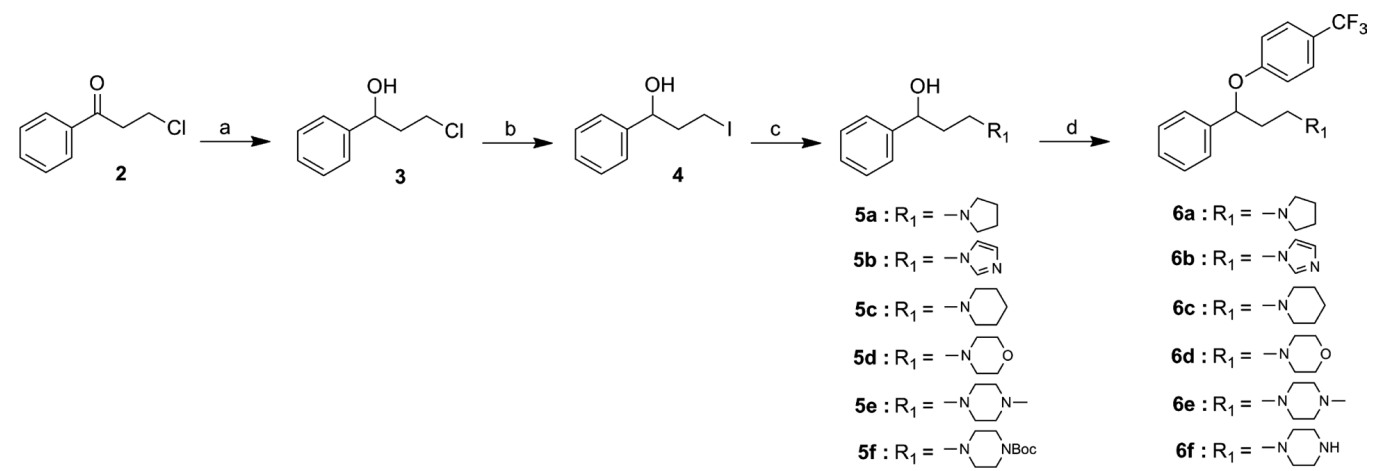

Reagents and conditions: (a) $\mathrm{NaBH}_{4}, \mathrm{MeOH}$, r.t., 24 h; (b) NaI, acetone, reflux, 48 h; (c) $\mathrm{R}_{1} \mathrm{H}$, THF, reflux, 4 h; and (d) NaH, DMA, p-chlorobenzotrifluoride, 70 to $100^{\circ} \mathrm{C}, 2 \mathrm{~h}$.

Chart 1

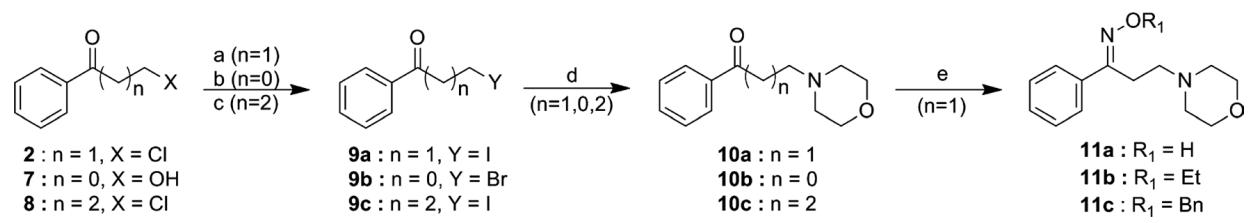

Reagents and conditions: (a) NaI, acetone, reflux, 4 h; (b) $\mathrm{CBr}_{4}, \mathrm{PPh}_{3}, \mathrm{ACN} / \mathrm{CH}_{2} \mathrm{Cl}_{2}$ (1/1), $0{ }^{\circ} \mathrm{C}, 15 \mathrm{~min}$; (c) NaI, acetone, reflux, $18 \mathrm{~h}$; (d) morpholine, THF, reflux, $4 \mathrm{~h}$; (e) $\mathrm{R}_{1}$ hydroxylamine hydrochloride, EtOH, pyridine, reflux, $1 \mathrm{~h}$.

Chart 2

romethyl phenyl ring was removed, were synthesized as depicted in Chart 2. Chloropropanone $\mathbf{2}$ was converted to iodopropanone 9a using $\mathrm{NaI}$ in acetone, which was then reacted with excess morpholine at reflux temperature for $4 \mathrm{~h}$ to give the crude residue containing product as well as excess morpholine. The desired morpholino-propanone 10a was obtained by chromatography on silica gel with dichloromethane/ methanol (v/v, 15/1). The various oxime analogues $(11 \mathrm{a}-\mathbf{c})$ were prepared by condensation reaction of $\mathbf{1 0 a}$ with corresponding hydroxylamine hydrochlorides.

To investigate the influence of the alkyl chain length in 10a on the activity, two different chain-length analogues $(\mathbf{1 0 b}, \mathbf{c})$ were synthesized as depicted in Chart 2, respectively. For the synthesis of $\mathbf{1 0 b}$, the alcohol 7 was first converted into the bromide 9b using triphenylphosphine $\left(\mathrm{PPh}_{3}\right)$ and tetrabromide $\left(\mathrm{CBr}_{4}\right)$ via an Appel reaction, and then reacted with excess morpholine to produce the crude product $\mathbf{1 0 b}$, which was purified by silica gel column chromatography to give pure 10b. Similarly, the crude product 10c was prepared from the conversion of 4-chlorobutanone $\mathbf{8}$ to its corresponding iodide 9c using $\mathrm{NaI}$ in acetone followed by the reaction of $9 \mathbf{c}$ with excess morpholine. Pure product 10c was obtained by column chromatography on silica gel.

Biological Effect of Fluoxetine Analogues Since LPS considerably induced NO production in BV2 cells and the level of NO production was detected by nitrite, in this study, fluoxetine and its analogues $(\mathbf{6} \mathbf{a}-\mathbf{f})$ were first evaluated for inhibitory effects on the production of NO in LPS-induced BV2 cells. After BV2 cells were co-treated with fluoxetine or its analogues at a concentration of $10 \mu \mathrm{M}$ and LPS (100 ng/ $\mathrm{ml}$ ) for $24 \mathrm{~h}$, the nitrite concentration was determined in the supernatant by the Griess assay, and their results are presented in Table 1. Among the fluoxetine analogues possessing various heterocyclic amine rings $(\mathbf{6 a}-\mathbf{f}), \mathbf{6 d}, \mathbf{6 e}$, and $\mathbf{6 f}$ showed inhibitory activities $(6.00 \pm 0.9 \%, 64.3 \pm 6.7 \%$ and
Table 1. Effects of Fluoxetine Heterocyclic Analogues on LPS-Induced NO Production

\begin{tabular}{|c|c|c|}
\hline Entry & Substituent $\left(\mathrm{R}_{1}\right)$ & Inhibition of NO release (\%) at $10 \mu \mathrm{m}$ \\
\hline 1 & $\mathrm{NHCH}_{3}$ & $20.8 \pm 0.9$ \\
\hline $6 a$ & $-N D$ & 0 \\
\hline $6 \mathrm{~b}$ & $-N=N$ & 0 \\
\hline $6 c$ & $-n$ & 0 \\
\hline 6d & & $6.00 \pm 0.9$ \\
\hline $6 e$ & & Cytotoxic $(64.3 \pm 6.7)$ \\
\hline $6 f$ & $-N$ & Cytotoxic $(79.6 \pm 2.3)$ \\
\hline
\end{tabular}

$79.6 \pm 2.3 \%$, respectively) comparable to that of fluoxetine $(20.8 \pm 0.9 \%)$, while the other heterocyclic analogues exhibited no activity at a concentration of $10 \mu \mathrm{M}$ in NO production.

To determine whether the inhibitory effects of fluoxetine and its analogues on NO production were related to their toxicity, the cytotoxicity of fluoxetine analogues was evaluated by MTT (3-[4,5-dimethylthiazol-2-yl]-2,5-diphenyltetrazolium bromide) assay. ${ }^{27)}$ As shown in Fig. 1, the relative cell viability of the BV2 cells treated with fluoxetine at the concentrations of $10 \mu \mathrm{M}, 20 \mu \mathrm{M}$ and $40 \mu \mathrm{M}$ was $52.3 \pm 3.4 \%$, $73.9 \pm 2.2 \%$ and $20.1 \pm 0.6 \%$, respectively, which indicates that fluoxetine did not suppress the cell viability of the BV2 cell at the concentrations of $10 \mu \mathrm{M}$ or $20 \mu \mathrm{M}$, but did exhibit cytotoxicity at the concentration of $40 \mu \mathrm{M}$. This result was consistent with a previous report. ${ }^{22)}$ The relative cell viability 


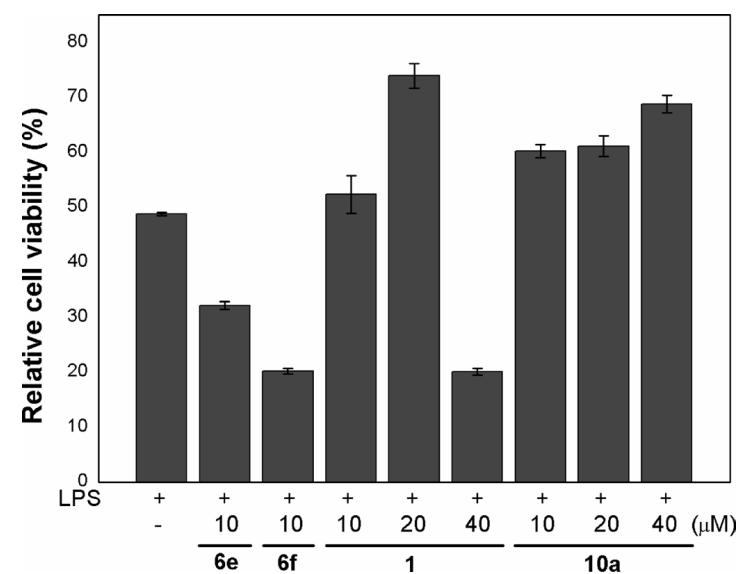

Fig. 1. Effect of Fluoxetine and Its Analogues on the Cell Viability of BV2 Cells

Cells $\left(2 \times 10^{5}\right.$ cells) were incubated with LPS $(1 \mu \mathrm{g} / \mathrm{ml})$ and each sample (fluoxetine and its analogues) for $24 \mathrm{~h}$. Cell viability was measured by MTT reduction assays and was expressed relative to the culture treated only with LPS (control; 48.8\%). Each value indicated the mean \pm S.E.M. and was representative of results obtained from three independent experiments.

of the BV2 cells treated with fluoxetine analogues $(\mathbf{6 e}, \mathbf{f})$ was also decreased to $32.1 \pm 0.7 \%$ and $20.2 \pm 0.5 \%$, respectively (Fig. 1), which clearly indicated that the inhibitory effect on NO production of the fluoxetine analogues $(6 \mathbf{e}, \mathbf{f})$ was due to their cytotoxicity rather than their intrinsic activities. In contrast, $6 \mathbf{d}$ containing the morpholine moiety did not show any cytotoxicity even at the concentration of $40 \mu \mathrm{M}$ (data not shown), despite of its weak inhibitory activity in NO production. Due to the absence of any cytotoxic effect, $\mathbf{6 d}$ was selected as a lead compound for further modification study.

The structure of $\mathbf{6} \mathbf{d}$ was simplified by removing the trifluoromethyl phenyl ring. Various functional moieties such as hydroxyl, ketone and oxime groups were introduced for replacing the ether linkage in fluoxetine while the morpholine moiety was fixed. The simplified compounds were tested for their inhibitory effects in NO production on BV2 cells. As shown in Table 2, none of the tested compounds (5d, 11ac) exhibited any inhibitory effect in NO production on BV2 cells, except 10a containing a ketone group. 10a revealed almost the same inhibitory activity as that of fluoxetine at the concentration of $10 \mu \mathrm{M}$. When we tested the inhibitory activities at different concentrations, 10a reduced the LPS-induced nitrite production in a dose-dependent manner. Accordingly, the percentage of inhibited NO production in 10a was $22.1 \pm 4.8,35.2 \pm 3.4$ and $83.7 \pm 1.4 \%$ at the concentrations of 10, 20 and $40 \mu \mathrm{M}$, respectively (Table 2).

To confirm that the suppressed effect of 10a on NO production was not caused by cytotoxicity, the cell viability was measured. LPS dramatically increased the concentration of nitrite from the cells $(47.1 \pm 2.3 \mu \mathrm{M})$ whereas a small amount of NO was produced $(5.47 \pm 1.22 \mu \mathrm{M})$ in unstimulated BV2 cells. The relative cell viability of the BV2 cells treated with $10 \mathrm{a}$ at the concentrations of $10 \mu \mathrm{M}, 20 \mu \mathrm{M}$ and $40 \mu \mathrm{M}$ was $60.2 \pm 1.2 \%, 61.1 \pm 1.9 \%$ and $68.8 \pm 1.6 \%$, respectively (Fig. 1 ), which indicated that 10a did not exhibit any cytotoxicity even at the concentration of $40 \mu \mathrm{m}$, while fluoxetine at the same concentration did. 10a also exhibited NO inhibitory activity in a dose-dependent manner (Fig. 2).

To investigate the influence of the alkyl chain length be-
Table 2. Effects of Simplified Fluoxetine Analogues on LPS-Induced NO Production

\begin{tabular}{cccc} 
& & \\
\hline \hline Entry & Substituent $\left(\mathrm{R}_{1}\right)$ & $n$ & Inhibition of $\mathrm{NO}$ release $(\%)$ at $10 \mu \mathrm{M}$ \\
\hline $\mathbf{1}$ & Fluoxetine & $20.8 \pm 0.9$ \\
& & & 0 \\
$\mathbf{5 d}$ & $-\mathrm{OH}$ & 1 & $22.0 \pm 1.1(20 \mu \mathrm{M})$ \\
$\mathbf{1 0}$ & $=\mathrm{O}$ & 1 & $35.2 \pm 3.4(20 \mu \mathrm{M})$ \\
& & & $83.7 \pm 1.4(40 \mu \mathrm{M})$ \\
$\mathbf{1 0 b}$ & $=\mathrm{O}$ & 0 & 0 \\
$\mathbf{1 0 c}$ & $=\mathrm{O}$ & 2 & 0 \\
$\mathbf{1 1}$ & $=\mathrm{N}-\mathrm{OH}$ & 1 & 0 \\
$\mathbf{1 1 b}$ & $=\mathrm{N}-\mathrm{OEt}$ & 1 & 0 \\
$\mathbf{1 1 c}$ & $=\mathrm{N}-\mathrm{OBn}$ & 1 & \\
& & &
\end{tabular}

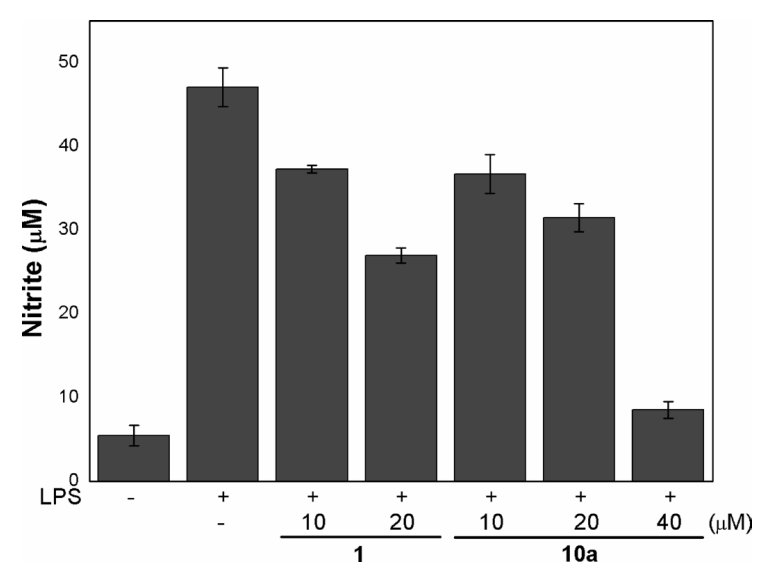

Fig. 2. Effects of Fluoxetine and 10a on LPS-Induced NO Production in BV2 Cells

BV2 cells were stimulated with LPS $(100 \mathrm{ng} / \mathrm{ml})$, co-treated with LPS $(100 \mathrm{ng} / \mathrm{ml})$ and fluoxetine at a concentrations of 10 and $20 \mu \mathrm{M}$ or co-treated with LPS $(100 \mathrm{ng} / \mathrm{ml})$ and 10a at a concentrations of 10,20 and $40 \mu \mathrm{m}$ for $24 \mathrm{~h}$. The amounts of NO were determined using the Griess reagent. All the data of each group are expressed as the mean \pm S.E.M. and are representative of results obtained from three independent experiments.

tween the ketone group and the morpholine moiety in 10a on the activity, two analogues $(\mathbf{1 0 b}, \mathbf{c})$ were also evaluated for the NO production activities in BV2 cells. Interestingly, these two analogues did not exhibit any effect at a concentration of $10 \mu \mathrm{M}$, which implied that the chain length between the oxo group and the heteroamine moiety plays an important role in maintaining the activity.

NO is biosynthesized by NOS, including, for example, endothelial NOS (eNOS), neuronal NOS (nNOS) and iNOS. The iNOS-derived NO is generated from various cells such as macrophages, monocytes and microglial cells and the overproduction of NO is caused by inflammation. Therefore, the reduced NO production is due to the inhibited iNOS expression. To examine whether the NO production was caused by a decrease in the mRNA level of iNOS, LPS-stimulated BV2 cells were treated with 10a and then the amount of iNOS was measured by reverse transcription polymerase chain reaction (RT-PCR). The expression of iNOS mRNA in LPS-treated BV2 cells was significantly increased in comparison with the non-treated control cells. When the LPS-treated BV2 cells were treated with $\mathbf{1 0 a}$ at different concentrations 

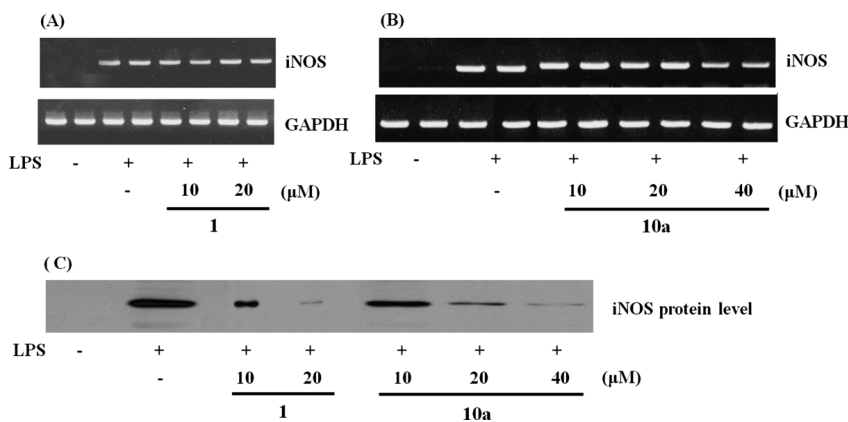

Fig. 3. Effects of Fluoxetine and 10a on iNOS Production in LPS-Induced BV2 Cells

BV2 cells were treated with LPS $(100 \mathrm{ng} / \mathrm{ml}$ or $200 \mathrm{ng} / \mathrm{ml})$ and in the absence or presence of fluoxetine and 10a. The expression levels of iNOS mRNA in fluoxetine (A) and 10a (B) were determined by RT-PCR. The production of iNOS protein level in fluoxetine and 10a $(\mathrm{C})$ was determined by immunoblotting with antibody iNOS.

$(10,20,40 \mu \mathrm{M})$, the cells were decreased to the density of iNOS mRNA in a dose-dependent manner (Figs. 3A, B). To confirm the effects of 10a on iNOS protein expression, the amount of iNOS protein expression was measured by the immunoblotting method. As shown in Fig. 3C, no iNOS protein was detected in unstimulated BV2 cells, whereas iNOS protein was induced in LPS-stimulated BV2 cells. When the LPS-treated BV2 cells were treated with 10a at different concentrations $(10,20,40 \mu \mathrm{M})$, the densities of iNOS protein in the cells were decreased in a dose-dependent manner. These results indicated that the inhibitory action of $10 \mathbf{a}$ on NO production was related to a modulation of iNOS induction.

10a was shown to have weak activities against the EMT6 tumor in vitro and against ADP-induced aggregation of human blood platelets in vitro, ${ }^{28,29)}$ while there was no effect in inhibition of edema in the test for anti-inflammatory activity in $\mathrm{CF}_{1}$ mice at $8 \mathrm{mg} / \mathrm{kg}$ as a series of $\beta$-alkylaminoketones. ${ }^{30)}$ However, there is no previous report regarding the suppression of NO production by 10a in LPS-induced BV2 cells.

In conclusion, by modifying the structure of fluoxetine, we discovered an analogue of fluoxetine 10a, which inhibited not only NO production but also iNOS expression in LPSinduced BV2 cells without much cytotoxicity. The trifluoromethyl phenyl moiety was not essential for the suppression in NO production. The simplified structure of $\mathbf{1 0 a}$ can be used for the further development of potent inhibitors of NO production.

Acknowledgments This study was financially supported by SH pharmaceutical company and the Post Brain Korea 21 program.

\section{REFERENCES}

1) Meng X. L., Yang J. Y., Chen G. L., Zhang L. J., Wang L. H., Li J., Wang J. M., Wu C. F., Int. Immunopharmacol., 8, 1074-1082 (2008).

2) Blaylock R. L., Journal of American Physicians and Surgeons, 9, 4651 (2004).

3) Takeuchi H., Mizuno T., Zhang G., Wang J., Kawanokuchi J., Kuno R., Suzumura A., J. Biol. Chem., 280, $10444-10454$ (2005).

4) Gehrmann J., Matsumoto Y., Kreutzberg G. W., Brain Res. Rev., 20, 269-287 (1995).

5) Banati R. B., Gehrmann J., Schubert P., Kreutzberg G. W., Glia, 7, $111-118$ (1993).

6) Moon D., Choi Y. H., Kim N., Park Y., Kim G., Int. Immunopharmacol., 7, 506-514 (2007).

7) Jin C., Moon D., Lee K., Kim M., Lee J., Choi Y. H., Park Y., Kim G., Pharmacol. Res., 54, 461-467 (2006).

8) Jantzen P. T., Connor K. E., Dicarlo G., Wenk G. L., Wallace J. L., Rojiani A. M., Coppola D., Morgan D., Gordon M. N., J. Neurosci., 22, 2246-2254 (2002)

9) Takeuchi H., Wang J., Kawanokuchi J., Mitsuma N., Mizuno T., Suzumura A., Neurobiol. Dis., 22, 33-39 (2006).

10) Chen R., Chen T., Chen T., Lin L., Chang C., Chang H., Wu C., Ann. N.Y. Acad. Sci., 1042, 262-271 (2005).

11) Aktan F., Life Sci., 75, 639-653 (2004).

12) Rafi M. M., Yadav P. N., Reyes M., J. Food Sci., 72, S69-S74 (2007).

13) Cui Y., Zhang L., Zhang T., Luo D., Jia Y., Guo Z., Zhang Q., Wang X., Wang X., Clin. Exp. Pharmacol. Physiol., 37, 422 - 428 (2010).

14) Jung K. K., Lee H. S., Cho J. Y., Shin W. C., Rhee M. H., Kim T. G., Kang J. H., Kim S. H., Hong S., Kang S. Y., Life Sci., 79, 2022-2031 (2006).

15) Park Y., Koketsu M., Kim J. M., Yeo J., Ishihara H., Lee K., Kim S. Y., Kim C., Biol. Pharm. Bull., 26, 1657-1660 (2003).

16) Hou R. C., Chen H., Tzen J. T. C., Jeng K. G., Neuro Report, 14, 1815-1819 (2003).

17) Choi S., Park Y., Choi D., Chang H., J. Microbiol. Biotechnol., 18, 1990-1996 (2008)

18) Abdel-Salam O. M. E., Baiuomy A. R., Arbid M. S., Pharmacol. Res., 49, 119-131 (2004).

19) Wilde M. I., Benfield P., Pharmacoeconomics, 13, 543-561 (1998).

20) Zafir A., Banu N., Eur. J. Pharmacol., 572, 23-31 (2007).

21) Mostert J. P., Koch M. W., Heerings M., Heersema D. J., Keyser J. D., CNS Neurosci. Ther., 14, 153-164 (2008).

22) Lim C., Kim S., Park J., Kim C., Yoon S., Lee J., J. Neurosci. Res., 87, 1037-1045 (2009)

23) Synder H. R., Brewster J. M., J. Am. Chem. Soc., 70, 4230-4232 (1948).

24) Yu Y., Kim J., Lee K., Kim S. Y., Han P., Lee J., Stroke, 36, 2238$2243(2005)$

25) Kim S., Jeong J., Kim H. J., Seo J., Han P., Yoon S., Lee J., Neurotox. Res., 17, 39-49 (2010).

26) Robertson D. W., Krushinski J. H., Fuller R. W., Leander J. D., J. Med. Chem., 31, 1412-1417 (1988).

27) Fotakis G., Timbrell J. A., Toxicol. Lett., 10, 171-177 (2006)

28) Dimmock J. R., Erciyas E., Raghavan S. K., Kirkpatrick D. L., Pharmazie, 45, 755-757 (1990).

29) Cazin M., Lesieur I., Boniface B., Boniface M., Lesieur D., Cazin J. C., Acta Therapeutica, 6, 205-211 (1980).

30) Huang Y., Hall I. H., Res. Commun. Pharmacol. Toxicol., 1, 17-38 (1996). 